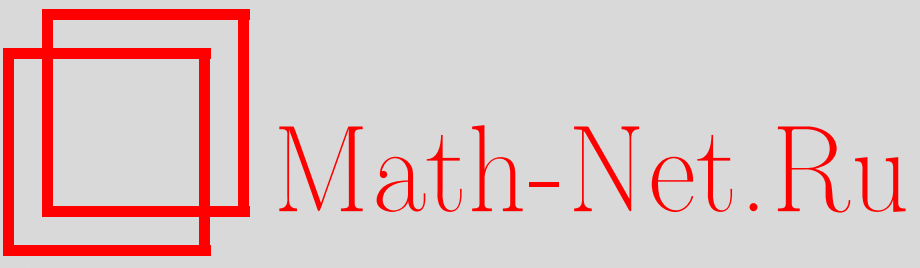

M. I. Gomoyunov, D. A. Serkov, On guarantee optimization in control problem with finite set of disturbances, Vestn. Udmurtsk. Univ. Mat. Mekh. Komp. Nauki, 2021, Volume 31, Issue 4, 613-628

DOI: https://doi.org/10.35634/vm210406

Use of the all-Russian mathematical portal Math-Net.Ru implies that you have read and agreed to these terms of use

http://www.mathnet.ru/eng/agreement

Download details:

IP: 34.229 .108 .108

April 26, 2023, 12:45:03 
MSC2020: 49N30, 49N35, 93C15

\author{
(C) M. I. Gomoyunov, D. A. Serkov
}

\title{
ON GUARANTEE OPTIMIZATION IN CONTROL PROBLEM WITH FINITE SET OF DISTURBANCES
}

In this paper, we deal with a control problem under conditions of disturbances, which is stated as a problem of optimization of the guaranteed result. Compared to the classical formulation of such problems, we assume that the set of admissible disturbances is finite and consists of piecewise continuous functions. In connection with this additional functional constraint on the disturbance, we introduce an appropriate class of non-anticipative control strategies and consider the corresponding value of the optimal guaranteed result. Under a technical assumption concerning a property of distinguishability of the admissible disturbances, we prove that this result can be achieved by using control strategies with full memory. As a consequence, we establish unimprovability of the class of full-memory strategies. A key element of the proof is a procedure of recovering the disturbance acting in the system, which allows us to associate every non-anticipative strategy with a full-memory strategy providing a close guaranteed result. The paper concludes with an illustrative example.

Keywords: control problem under disturbances, optimal guaranteed result, non-anticipative strategy, fullmemory strategy, recovery of disturbances, unimprovability.

DOI: $10.35634 / \mathrm{vm} 210406$

\section{Introduction}

The paper deals with a control problem under conditions of disturbances (see, e.g., [1-4]) involving a dynamical system described by a non-linear ordinary differential equation and a cost functional, which evaluates system's motions. The aim of the control is to minimize the value of the cost functional against the worst case disturbances. So, the control problem is stated as a problem of optimization of the guaranteed result. In the classical formulation of such problems, it is usually assumed (see, e.g., [3, Section 1.4], [4, Section 6], and also [5]) that the set of admissible disturbances, similarly to the set of admissible controls, consists of all measurable functions with values in a given set, describing instantaneous (geometric) constraints. In this paper, we continue the research initiated in $[6,7]$ and focus on the case when the set of admissible disturbances is more specific, that is, there are additional so-called functional constraints on the disturbance (for some examples, see, e.g., [8-12]).

In [7], to cope with the features arising from the presence of such functional constraints, it was suggested to modify in an appropriate way the standard notion of a non-anticipative control strategy (see, e.g., $[13,14]$ ) and investigate the guarantee optimization problem in this new class of strategies. The main result of the present paper is the proof of the fact that, under some additional assumptions, the corresponding value of the optimal guaranteed result can be achieved with the help of feedback control strategies, or, more precisely, control strategies with full memory (see, e.g., [5] and also [15]), which are acceptable from a practical point of view. In particular, this result implies that the value of the optimal guaranteed result in the class of non-anticipative strategies coincides with that in the class of full-memory strategies, which means that the class of full-memory strategies is unimprovable in the sense of [5] (see also [15]).

Compared to the general formulation of the problem presented in [7], we assume that the dynamics of the system is separated with respect to control and disturbance actions and the set of 
admissible disturbances is finite and consists of piecewise continuous functions. Let us note that control problems with a finite set of disturbances have many practical applications. For instance, such problems can be viewed as control problems under uncertainty described by a finite set of scenarios of evolution of some system parameters (see, e.g., [10]).

Let us briefly discuss some of the peculiarities of the problem considered in the paper. First, the problem differs from so-called minimax optimal control problems (see, e.g., [16]) in which optimization is performed over the class of open-loop (program) controls. Indeed, it can happen that the value of the optimal guaranteed result in the class of open-loop controls is greater than that in the class of feedback control strategies. Second, in contrast to, e.g., [12], we do not require a possibility to recover the disturbance during a short initial period of time by observing the system's motion, which would allow us, on the remaining time interval, substitute this disturbance into the dynamics and solve the corresponding optimal control problem (without any uncertainty). In fact, we do not exclude the situations when, during the whole control process, we cannot find out exactly which one of the admissible disturbances is acting in the system, and, therefore, we must choose current control actions under conditions of incomplete information about the future behavior of the system. Third, in general, we could pass from the considered problem to the corresponding guarantee optimization problem in which all measurable disturbances satisfying the instantaneous constraints are admissible. In particular, this would allow us to directly use the approaches and methods developed in the theory of positional differential games (see, e.g., [3,4]). However, it can happen that a feedback (namely, positional) control strategy that is optimal in the obtained extended problem does not guarantee the desired result in the original problem with the finite set of disturbances. Finally, let us note also that all the peculiarities described above can be readily seen in the example presented in Section 5 below.

In addition, let us make some remarks concerning the proof of the main result of the paper. Let us recall that, according to the technique provided by the theory of positional differential games, a usual way of how to built a feedback control strategy that guarantees a given value of the cost functional against every admissible disturbance is to construct an appropriate so-called $u$-stable set or $u$-stable function and then apply the corresponding procedure of extremal aiming (see, e.g., [3, Sections 2.2 and 2.4] and [4, Section 8]). Trying to follow this direction in the considered problem with the finite set of disturbances, we could take, for instance, the $u$-stable optimal guaranteed result function proposed in [7], but, nevertheless, certain difficulties would be encountered in designing of an appropriate extremal aiming procedure. The reason for these difficulties lies primarily in the fact that the set of all the disturbances that remain admissible up to a current time depends on the history of the system's motion.

In this connection, in order to show that the value of the optimal guaranteed result in the class of non-anticipative control strategies is achieved in the class of control strategies with full memory, we adopt another approach. Namely, we propose a procedure that associates every nonanticipative strategy with a full-memory strategy such that the following property holds: for any admissible disturbance, the system's motions generated by the non-anticipative strategy and by the full-memory strategy are close to each other with respect to the uniform norm. Hence, the associated full-memory strategy guarantees the value of the cost functional close to that guaranteed by the original non-anticipative strategy, and the desired statement follows. A key element in the construction of the full-memory strategy is a rule that, during the control process, recovers the set of all the admissible disturbances that, together with test controls assigned at certain periods of time, agree with the observed system's motion. The successful identification of this set allows us to predict the future values of the unknown disturbance acting in the system and, therefore, makes it possible to use the corresponding controls assigned by the original non-anticipative strategy. It should be emphasized that the correctness of the arguments described above is established in the paper under an additional assumption, concerning some special property of distinguishability 
of the admissible disturbances by observing the corresponding system's motions. In spite of the fact that this assumption narrows the applicability of the result, it is valid, for instance, if the right-hand side of the dynamic equation is injective with respect to disturbance actions and all the admissible disturbances are piecewise constant.

The paper is organized as follows. In Section 1, we formulate the considered control problem under conditions of disturbances. In particular, we describe the dynamical system, the sets of admissible controls and disturbances, and the cost functional. In Section 2, we introduce the classes of non-anticipative strategies and full-memory strategies and establish a connection between them. In Section 3, we show how to construct the full-memory strategy associated with a given non-anticipative strategy. In Section 4, we prove the main result and present a class of problems for which all the assumptions made in the paper are satisfied. In Section 5, we provide an illustrative example.

\section{$\S 1$. Statement of the problem}

We consider a dynamical system whose motion is described by the differential equation

$$
d x(t) / d t=f(t, x(t), u(t))+g(t, x(t), v(t)), \quad t \in T:=\left[t_{0}, \vartheta\right]
$$

and the initial condition

$$
x\left(t_{0}\right)=x_{0}
$$

Here, $t$ is time; $x(t) \in \mathbb{R}^{n}$ is a current value of the state vector; $u(t) \in \mathbb{R}^{p}$ and $v(t) \in \mathbb{R}^{q}$ are current values of the control and disturbance, respectively; $x_{0} \in \mathbb{R}^{n}$ is a fixed initial value of the state vector. The control and disturbance are subject to the instantaneous constraints

$$
u(t) \in P, \quad v(t) \in Q, \quad t \in T,
$$

where $P \subset \mathbb{R}^{p}$ and $Q \subset \mathbb{R}^{q}$ are given compact sets. The functions $f: T \times \mathbb{R}^{n} \times P \rightarrow \mathbb{R}^{n}$ and $g: T \times \mathbb{R}^{n} \times Q \rightarrow \mathbb{R}^{n}$ are continuous and satisfy a local Lipschitz continuity and a sublinear growth conditions with respect to the second variable (see, e.g., [3, Section 1.2] and [4, Section 3] for details).

Let $\mathcal{U}$ and $\mathcal{V}$ be the sets of all (Lebesgue) measurable functions $u: T \rightarrow P$ and $v: T \rightarrow Q$, respectively. In accordance with (1.3), by an admissible control, we mean every function $u(\cdot) \in$ $\in \mathcal{U}$. On the other hand, we suppose that a finite set

$$
V:=\left(v_{\ell}(\cdot)\right)_{\ell \in \overline{1, n_{V}}} \subset \mathcal{V}
$$

is specified and only the functions $v_{\ell}(\cdot), \ell \in \overline{1, n_{V}}$, are admissible disturbances. Thus, in the terminology of [7], the disturbance is subject to the additional functional constraint $v(\cdot) \in V$. Moreover, we assume that every function $v_{\ell}(\cdot), \ell \in \overline{1, n_{V}}$, is piecewise continuous and

$$
v_{\ell}\left(t_{0}\right)=v_{m}\left(t_{0}\right), \quad \ell, m \in \overline{1, n_{V}} .
$$

Let us note that, throughout the paper, we call a function defined on $T$ piecewise continuous if it is left-continuous, has at most a finite number of discontinuity points, and, at every discontinuity point, possesses a finite right limit.

Due to the conditions imposed on the functions $f$ and $g$, for every $u(\cdot) \in \mathcal{U}$ and every $v(\cdot) \in \mathcal{V}$, there exists a unique system's motion $x(\cdot):=x(\cdot ; u(\cdot), v(\cdot))$, which is an absolutely continuous function $x: T \rightarrow \mathbb{R}^{n}$ that meets initial condition (1.2) and, together with $u(\cdot)$ and $v(\cdot)$, 
satisfies differential equation (1.1) for almost every (a.e.) $t \in T$. In addition, we can choose numbers $R>0$ and $L>0$ such that

$$
\|x(t ; u(\cdot), v(\cdot))\| \leq R, \quad\|x(t ; u(\cdot), v(\cdot))-x(\tau ; u(\cdot), v(\cdot))\| \leq L|t-\tau|
$$

for any $t, \tau \in T$, any $u(\cdot) \in \mathcal{U}$, and any $v(\cdot) \in \mathcal{V}$, and find a number $\lambda>0$ such that

$$
\|f(t, x, u)-f(t, y, u)\|+\|g(t, x, v)-g(t, y, v)\| \leq \lambda\|x-y\|
$$

for any $t \in T$, any $x, y \in \mathbb{R}^{n}$ with $\|x\| \leq R,\|y\| \leq R$, any $u \in P$, and any $v \in Q$.

We assume that a cost functional has the form

$$
J\left(u(\cdot), v_{\ell}(\cdot)\right)=\sigma\left(x\left(\cdot ; u(\cdot), v_{\ell}(\cdot)\right)\right), \quad u(\cdot) \in \mathcal{U}, \quad \ell \in \overline{1, n_{V}} .
$$

Here, $\sigma: C\left(T ; \mathbb{R}^{n}\right) \rightarrow \mathbb{R}$ is a continuous function, where $C\left(T ; \mathbb{R}^{n}\right)$ is the space of all continuous functions from $T$ to $\mathbb{R}^{n}$ endowed with the uniform norm. The aim of the control is to minimize the value of the cost functional against the worst case admissible disturbances. Thus, the control problem under consideration is stated as a guarantee optimization problem.

\section{$\S 2$. Control strategies}

Since $u(t)$ and $v(t)$ are separated in differential equation (1.1) and the set $V$ of admissible disturbances is finite and consists of piecewise continuous functions (see (1.4)), the non-anticipative (control) strategies introduced in [7, Section 1.4] can be defined as follows.

Consider a mapping $\alpha: V \rightarrow \mathcal{U}$. Put

$$
u_{\ell}(\cdot):=\alpha\left(v_{\ell}(\cdot)\right)(\cdot), \quad x_{\ell}(\cdot):=x\left(\cdot ; u_{\ell}(\cdot), v_{\ell}(\cdot)\right), \quad \ell \in \overline{1, n_{V}},
$$

and, for any $\ell, m \in \overline{1, n_{V}}$ and any $t \in T$, denote

$$
f_{\ell, m}(t):=f\left(t, x_{\ell}(t), u_{m}(t)\right), \quad f_{\ell}(t):=f_{\ell, \ell}(t)
$$

and

$$
g_{\ell, m}(t):=g\left(t, x_{\ell}(t), v_{m}(t)\right), \quad g_{\ell}(t):=g_{\ell, \ell}(t) .
$$

Then, the mapping $\alpha$ is called a non-anticipative strategy if, for every $\ell, m \in \overline{1, n_{V}}$ and every $s \in\left(t_{0}, \vartheta\right]$ satisfying the condition

$$
g_{\ell}(t)=g_{\ell, m}(t), \quad t \in\left[t_{0}, s\right]
$$

it holds that

$$
x_{\ell}(t)=x_{m}(t), \quad t \in\left[t_{0}, s\right] .
$$

Observe that, in view of (1.1), equalities (2.4) and (2.5) imply that

$$
f_{\ell}(t)=f_{\ell, m}(t) \quad \text { for a.e. } t \in\left[t_{0}, s\right] .
$$

Let $\mathcal{A}$ be the set of all non-anticipative strategies. Respectively, the value of the optimal guaranteed result in the class of non-anticipative strategies is given by

$$
\Gamma:=\inf _{\alpha \in \mathcal{A}} \max _{\ell \in \overline{1, n_{V}}} \sigma\left(x\left(\cdot ; \alpha\left(v_{\ell}(\cdot)\right)(\cdot), v_{\ell}(\cdot)\right)\right) .
$$


Further, following, e.g., [5,15], we introduce a notion of a full-memory (control) strategy.

A set $\Delta:=\left(\tau_{i}\right)_{i \in \overline{0, n_{\Delta}}} \subset T$ such that $\tau_{0}=t_{0}, \tau_{i-1}<\tau_{i}$ for all $i \in \overline{1, n_{\Delta}}$, and $\tau_{n_{\Delta}}=\vartheta$ is called a partition of the interval $T$. The set of all such partitions is denoted by $\Delta_{T}$. A full-memory feedback on a partition $\Delta \in \Delta_{T}$ is a family $U^{\Delta}:=\left(U_{i}^{\Delta}\right)_{i \in \overline{0, n_{\Delta}-1}}$ of mappings

$$
U_{i}^{\Delta}:\left.C\left(\left[t_{0}, \tau_{i}\right] ; \mathbb{R}^{n}\right) \rightarrow \mathcal{U}\right|_{\left[\tau_{i}, \tau_{i+1}\right)}, \quad i \in \overline{0, n_{\Delta}-1},
$$

where $\left.\mathcal{U}\right|_{\left[\tau_{i}, \tau_{i+1}\right)}$ is the set of restrictions of all functions $u(\cdot) \in \mathcal{U}$ to $\left[\tau_{i}, \tau_{i+1}\right)$. A full-memory feedback $U^{\Delta}$ in a pair with an admissible disturbance $v_{\ell}(\cdot), \ell \in \overline{1, n_{V}}$, forms in system (1.1), (1.2) a control $u(\cdot) \in \mathcal{U}$ according to the step-by-step rule

$$
u(t):=U_{i}^{\Delta}\left(\left.x(\cdot)\right|_{\left[t_{0}, \tau_{i}\right]}\right)(t), \quad t \in\left[\tau_{i}, \tau_{i+1}\right), \quad i \in \overline{0, n_{\Delta}-1},
$$

where $\left.x(\cdot)\right|_{\left[t_{0}, \tau_{i}\right]}$ is the restriction of the system's motion $x(\cdot)$ to $\left[t_{0}, \tau_{i}\right]$. For definiteness, we formally put $u(\vartheta):=u^{*}$ for a fixed $u^{*} \in P$. Let $x\left(\cdot ; U^{\Delta}, v_{\ell}(\cdot)\right)$ denote the system's motion uniquely generated by $U^{\Delta}$ and $v_{\ell}(\cdot)$.

By a full-memory strategy, we mean a family $U:=\left(U^{\Delta}\right)_{\Delta \in \Delta_{T}}$ of full-memory feedbacks specified for all partitions $\Delta \in \Delta_{T}$. Let $\mathcal{S}$ be the set of all full-memory strategies. The value of the optimal guaranteed result in the class of full-memory strategies is defined by

$$
\Gamma_{*}:=\inf _{U \in \mathcal{S}} \lim _{\delta \rightarrow+0} \sup _{\Delta \in \Delta_{T}: \mathrm{d}(\Delta) \leq \delta} \max _{\ell \in \overline{1, n_{V}}} \sigma\left(x\left(\cdot ; U^{\Delta}, v_{\ell}(\cdot)\right)\right),
$$

where $\mathrm{d}(\Delta):=\max _{i \in \overline{1, n_{\Delta}}}\left(\tau_{i}-\tau_{i-1}\right)$ is the diameter of the partition $\Delta$.

Lemma 1. The optimal guaranteed result in the class of non-anticipative strategies is not greater than that in the class of full-memory strategies, i.e., the inequality $\Gamma \leq \Gamma_{*}$ holds.

P r o o f. Fix $U:=\left(U^{\Delta}\right)_{\Delta \in \Delta_{T}} \in \mathcal{S}$ and $\Delta:=\left(\tau_{i}\right)_{i \in \overline{0, n_{\Delta}}} \in \Delta_{T}$. For every $\ell \in \overline{1, n_{V}}$, consider the motion $x_{\ell}(\cdot):=x\left(\cdot ; U^{\Delta}, v_{\ell}(\cdot)\right)$ of system (1.1), (1.2) and the corresponding control, which is denoted by $u_{\ell}(\cdot)$. Define a mapping $\alpha: V \rightarrow \mathcal{U}$ by

$$
\alpha\left(v_{\ell}(\cdot)\right)(t):=u_{\ell}(t), \quad t \in T, \quad \ell \in \overline{1, n_{V}} .
$$

Let us show that $\alpha \in \mathcal{A}$, i. e., $\alpha$ is a non-anticipative strategy.

For every $\ell, m \in \overline{1, n_{V}}$, consider the auxiliary functions $f_{\ell, m}(\cdot), f_{\ell}(\cdot)$ and $g_{\ell, m}(\cdot), g_{\ell}(\cdot)$ given by (2.2) and (2.3).

Fix $\ell, m \in \overline{1, n_{V}}$ and $s \in\left(t_{0}, \vartheta\right]$ such that condition (2.4) holds. Let us denote

$$
i_{*}:=\max \left\{i \in \overline{0, n_{\Delta}-1}: \tau_{i}<s\right\}
$$

and prove that

$$
x_{\ell}(t)=x_{m}(t), \quad t \in\left[t_{0}, \tau_{i_{*}}\right] .
$$

Due to initial condition (1.2), we have $x_{\ell}\left(t_{0}\right)=x_{m}\left(t_{0}\right)$. Arguing by induction, let us take $i \in \overline{0, i_{*}-1}$ and assume that equality (2.11) is fulfilled for every $t \in\left[t_{0}, \tau_{i}\right]$. Then, in view of (2.8), we derive

$$
u_{\ell}(t)=U_{i}^{\Delta}\left(\left.x_{\ell}(\cdot)\right|_{\left[t_{0}, \tau_{i}\right]}\right)(t)=U_{i}^{\Delta}\left(\left.x_{m}(\cdot)\right|_{\left[t_{0}, \tau_{i}\right]}\right)(t)=u_{m}(t), \quad t \in\left[\tau_{i}, \tau_{i+1}\right),
$$

and, therefore, $f_{\ell}(t)=f_{\ell, m}(t)$ for all $t \in\left[\tau_{i}, \tau_{i+1}\right)$. Hence, and by (2.4), we get

$$
x_{\ell}(t)=x_{\ell}\left(\tau_{i}\right)+\int_{\tau_{i}}^{t}\left(f_{\ell}(\tau)+g_{\ell}(\tau)\right) d \tau=x_{m}\left(\tau_{i}\right)+\int_{\tau_{i}}^{t}\left(f_{\ell, m}(\tau)+g_{\ell, m}(\tau)\right) d \tau, \quad t \in\left[\tau_{i}, \tau_{i+1}\right] .
$$


Consequently, for all $t \in\left[\tau_{i}, \tau_{i+1}\right]$, we have

$\left\|x_{\ell}(t)-x_{m}(t)\right\| \leq \int_{\tau_{i}}^{t}\left(\left\|f_{\ell, m}(\tau)-f_{m}(\tau)\right\|+\left\|g_{\ell, m}(\tau)-g_{m}(\tau)\right\|\right) d \tau \leq \lambda \int_{\tau_{i}}^{t}\left\|x_{\ell}(\tau)-x_{m}(\tau)\right\| d \tau$

where $\lambda$ is the number from (1.7). Thus, by Gronwall's inequality, we obtain $x_{\ell}(t)=x_{m}(t)$ for all $t \in\left[\tau_{i}, \tau_{i+1}\right]$. As a result, we conclude that equality (2.11) is valid for all $t \in\left[t_{0}, \tau_{i_{*}}\right]$.

Further, arguing similarly to the induction step above, it can be proved that $x_{\ell}(t)=x_{m}(t)$ for all $t \in\left[\tau_{i_{*}}, s\right]$, too. By virtue of (2.5), the inclusion $\alpha \in \mathcal{A}$ follows.

Now, recalling that $x\left(\cdot ; U^{\Delta}, v_{\ell}(\cdot)\right)=x\left(\cdot ; \alpha\left(v_{\ell}(\cdot)\right)(\cdot), v_{\ell}(\cdot)\right)$ for all $\ell \in \overline{1, n_{V}}$ by construction (see (2.10)), in accordance with definition (2.7) of $\Gamma$, we derive

$$
\max _{\ell \in \overline{1, n_{V}}} \sigma\left(x\left(\cdot ; U^{\Delta}, v_{\ell}(\cdot)\right)\right) \geq \Gamma .
$$

Since estimate (2.12) is fulfilled for any full-memory strategy $U:=\left(U^{\Delta}\right)_{\Delta \in \Delta_{T}} \in \mathcal{S}$ and any partition $\Delta \in \Delta_{T}$, in view of definition (2.9) of $\Gamma_{*}$, we get $\Gamma_{*} \geq \Gamma$.

\section{$\S 3$. Construction of full-memory control strategy}

Let a non-anticipative strategy $\alpha \in \mathcal{A}$ be fixed. Let us consider the corresponding controls $u_{\ell}(\cdot)$, motions $x_{\ell}(\cdot)$ of system (1.1), (1.2), as well as auxiliary functions $f_{\ell, m}(\cdot), f_{\ell}(\cdot)$ and $g_{\ell, m}(\cdot), g_{\ell}(\cdot)$ for all $\ell, m \in \overline{1, n_{V}}$, which are given by (2.1)-(2.3).

For every $\ell, m \in \overline{1, n_{V}}$, put

$$
s_{\ell, m}:=\max \left\{s \in T: g_{\ell}(t)=g_{\ell, m}(t), t \in\left[t_{0}, s\right]\right\} .
$$

Let us note that the set under the maximum sign is not empty. Indeed, due to (1.5), we have $g_{\ell}\left(t_{0}\right)=g_{\ell, m}\left(t_{0}\right)$, and, therefore, this set contains the point $t_{0}$. In addition, recalling that the functions $v_{\ell}(\cdot)$ and $v_{m}(\cdot)$ are piecewise continuous, we conclude that the functions $g_{\ell}(\cdot)$ and $g_{\ell, m}(\cdot)$ are piecewise continuous, which implies that the maximum is attained. Moreover, since the mapping $\alpha$ is non-anticipative (see (2.4)-(2.6)), we obtain

$$
x_{\ell}(t)=x_{m}(t), \quad t \in\left[t_{0}, s_{\ell, m}\right],
$$

and, consequently, the equalities below are valid:

$$
g_{\ell}(t)=g_{\ell, m}(t)=g_{m}(t)=g_{m, \ell}(t), \quad t \in\left[t_{0}, s_{\ell, m}\right]
$$

and

$$
f_{\ell}(t)=f_{\ell, m}(t)=f_{m}(t)=f_{m, \ell}(t) \quad \text { for a. e. } t \in\left[t_{0}, s_{\ell, m}\right] .
$$

In particular, based on relations (3.2)-(3.4), we can say that the disturbances $v_{\ell}(\cdot)$ and $v_{m}(\cdot)$ are "undistinguishable" until $s_{\ell, m}$. On the other hand, if $s_{\ell, m}<\vartheta$, then, according to (3.1), these disturbances $v_{\ell}(\cdot)$ and $v_{m}(\cdot)$ are "distinguishable" in an arbitrarily small right neighbourhood of $s_{\ell, m}$. Namely, it holds that

$$
\left.g_{\ell}(\cdot)\right|_{\left[s_{\ell, m}, t\right]} \neq\left. g_{\ell, m}(\cdot)\right|_{\left[s_{\ell, m}, t\right]}, \quad t \in\left(s_{\ell, m}, \vartheta\right] .
$$

Respectively, in what follows, we say that the property of strict distinguishability (of the admissible disturbances) is fulfilled for the strategy $\alpha$ if, for every $\ell, m \in \overline{1, n_{V}}$ such that $s_{\ell, m}<\vartheta$, the condition below is satisfied:

$$
\lim _{t \rightarrow s_{\ell, m}+0}\left\|g_{\ell}(t)-g_{\ell, m}(t)\right\|>0 .
$$


The limit in (3.5) exists because the functions $g_{\ell}(\cdot)$ and $g_{\ell, m}(\cdot)$ are piecewise continuous.

Further, let us introduce the set

$$
\Delta_{*}:=\left\{s_{\ell, m}: \ell, m \in \overline{1, n_{V}}\right\} \cup\left\{t_{0}\right\} .
$$

Let us observe that $\vartheta \in \Delta_{*}$ since $s_{\ell, \ell}=\vartheta$ for any $\ell \in \overline{1, n_{V}}$. Therefore, the set $\Delta_{*}$ is a partition of the interval $T$, i. e., $\Delta_{*} \in \Delta_{T}$. Let us arrange all the points of $\Delta_{*}$ in ascending order and denote them by $\xi_{k}$ for $k \in \overline{0, n_{\Delta_{*}}}$. Thus, we have

$$
\Delta_{*}=\left(\xi_{k}\right)_{k \in \overline{0, n_{\Delta_{*}}}} .
$$

Now, let us proceed with a description of how, based on the non-anticipative strategy $\alpha$, we construct a full-memory strategy $U:=\left(U^{\Delta}\right)_{\Delta \in \Delta_{T}} \in \mathcal{S}$. Let us take a partition $\Delta:=\left(\tau_{i}\right)_{i \in \overline{0, n_{\Delta}}} \in$ $\in \Delta_{T}$. In accordance with (2.9), we can confine ourselves to the case when $\mathrm{d}(\Delta)<\mathrm{d}\left(\Delta_{*}\right)$. For every $k \in \overline{0, n_{\Delta_{*}}-1}$, denote

$$
i_{k}:=\min \left\{i \in \overline{1, n_{\Delta}}: \tau_{i}>\xi_{k}\right\} .
$$

Let us define the full-memory feedback $U^{\Delta}:=\left(U_{i}^{\Delta}\right)_{i \in \overline{0, n_{\Delta}-1}}$ as follows. For $i=0$, we set

$$
U_{0}^{\Delta}(x(\cdot))(t):=u_{*}, \quad t \in\left[\tau_{0}, \tau_{1}\right), \quad x(\cdot) \in C\left(\left[t_{0}, \tau_{0}\right] ; \mathbb{R}^{n}\right),
$$

where $u_{*} \in P$ is a fixed test control. For every $i \in \overline{1, n_{\Delta}-1}$, we first find $k \in \overline{0, n_{\Delta_{*}}-1}$ such that $\tau_{i} \in\left(\xi_{k}, \xi_{k+1}\right]$. Then, for a given function $x(\cdot) \in C\left(\left[t_{0}, \tau_{i}\right] ; \mathbb{R}^{n}\right)$, we choose

$$
\ell_{k}:=\min \left\{\underset{\ell \in \overline{1, n_{V}}}{\operatorname{Argmin}} \max _{j \in \overline{0, k}}\left\|\frac{x\left(\tau_{i_{j}}\right)-x\left(\xi_{j}\right)}{\tau_{i_{j}}-\xi_{j}}-\frac{1}{\tau_{i_{j}}-\xi_{j}} \int_{\xi_{j}}^{\tau_{i_{j}}}\left(f\left(t, x(t), u_{*}\right)+g_{\ell}(t)\right) d t\right\|\right\} .
$$

Note that $\tau_{i} \geq \tau_{i_{k}}$ and, therefore, the values $x(t)$ used in (3.9) are well-defined. After that, if $\tau_{i+1} \leq \xi_{k+1}$, we define

$$
U_{i}^{\Delta}(x(\cdot))(t):=u_{\ell_{k}}(t), \quad t \in\left[\tau_{i}, \tau_{i+1}\right)
$$

and, if $\tau_{i+1}>\xi_{k+1}$, which implies that $\tau_{i+1}=\tau_{i_{k+1}}$, we put

$$
U_{i}^{\Delta}(x(\cdot))(t):= \begin{cases}u_{\ell_{k}}(t), & \text { if } t \in\left[\tau_{i}, \xi_{k+1}\right) \\ u_{*}, & \text { if } t \in\left[\xi_{k+1}, \tau_{i+1}\right) .\end{cases}
$$

In other words, for every $k \in \overline{0, n_{\Delta_{*}}-1}$, the control on the interval $\left[\xi_{k}, \xi_{k+1}\right)$ is assigned by the following procedure. On the initial short period of time $\left[\xi_{k}, \tau_{i_{k}}\right)$, the test control $u_{*}$ is used. By observing the realized system's motion on all the test intervals $\left[\xi_{j}, \tau_{i_{j}}\right], j \in \overline{0, k}$, the admissible disturbance $v_{\ell_{k}}(\cdot)$ is determined according to the rule (3.9). On the remaining period of time $\left[\tau_{i_{k}}, \xi_{k+1}\right)$, the control $u_{\ell_{k}}(\cdot)$, specified by the non-anticipative strategy $\alpha$ for the disturbance $v_{\ell_{k}}(\cdot)$, is applied.

Lemma 2. Let a non-anticipative strategy $\alpha \in \mathcal{A}$ possess the property of strict distinguishability (see (3.5)). Let $U:=\left(U^{\Delta}\right)_{\Delta \in \Delta_{T}} \in \mathcal{S}$ be the full-memory strategy constructed on the basis of $\alpha$ by procedure (3.8)-(3.11). Then, for any $\eta>0$, there exists $\delta>0$ such that, for any partition $\Delta \in \Delta_{T}$ satisfying the condition $\mathrm{d}(\Delta) \leq \delta$ and any $\ell \in \overline{1, n_{V}}$, it holds that

$$
\left\|x\left(t ; U^{\Delta}, v_{\ell}(\cdot)\right)-x\left(t ; \alpha\left(v_{\ell}(\cdot)\right)(\cdot), v_{\ell}(\cdot)\right)\right\| \leq \eta, \quad t \in T .
$$


P r o o f. Let us note that, in the proof, the notation introduced above is used in relation to the given non-anticipative strategy $\alpha$.

The property of strict distinguishability implies that there is a number $\zeta>0$ such that, for any $\ell, m \in \overline{1, n_{V}}$ satisfying the condition $s_{\ell, m}<\vartheta$, the inequality below is valid:

$$
\lim _{t \rightarrow s_{\ell, m}+0}\left\|g_{\ell}(t)-g_{\ell, m}(t)\right\| \geq \zeta .
$$

Hence, recalling that the functions $g_{\ell}(\cdot)$ and $g_{\ell, m}(\cdot)$ for all $\ell, m \in \overline{1, n_{V}}$ are piecewise continuous, we can find $\omega \in\left(0, \mathrm{~d}\left(\Delta_{*}\right)\right)$ such that, for every $\ell, m \in \overline{1, n_{V}}$, if $s_{\ell, m}<\vartheta$, then

$$
\frac{1}{\tau-s_{\ell, m}}\left\|\int_{s_{\ell, m}}^{\tau}\left(g_{\ell}(t)-g_{\ell, m}(t)\right) d t\right\| \geq \frac{\zeta}{2}, \quad \tau \in\left(s_{\ell, m}, s_{\ell, m}+\omega\right] .
$$

Let $\eta>0$ be fixed. Choose $\delta>0$ satisfying the conditions

$$
\delta \leq \omega, \quad 2 L \lambda n_{\Delta_{*}} e^{\lambda\left(\vartheta-t_{0}\right)} \delta \leq \zeta / 8, \quad 2 L n_{\Delta_{*}} e^{\lambda\left(\vartheta-t_{0}\right)} \delta \leq \eta,
$$

where the numbers $L$ and $\lambda$ are taken from (1.6) and (1.7), respectively. Let us verify that the statement of the lemma holds for this number $\delta$.

Let $\Delta \in \Delta_{T}$ be such that $\mathrm{d}(\Delta) \leq \delta$, and let $\bar{\ell} \in \overline{1, n_{V}}$. Consider the system's motion $\bar{x}(\cdot):=x\left(\cdot ; U^{\Delta}, v_{\bar{\ell}}(\cdot)\right)$ and the corresponding control, which is denoted by $\bar{u}(\cdot)$. Put

$$
\bar{f}(t):=f(t, \bar{x}(t), \bar{u}(t)), \quad \bar{g}(t):=g\left(t, \bar{x}(t), v_{\bar{\ell}}(t)\right), \quad t \in T .
$$

Let us observe that, according to the definition of the full-memory feedback $U^{\Delta}$, for every $k \in \overline{0, n_{\Delta_{*}}-1}$, we obtain

$$
\bar{u}(t)= \begin{cases}u_{*}, & \text { if } t \in\left[\xi_{k}, \tau_{i_{k}}\right), \\ u_{\bar{\ell}_{k}}(t), & \text { if } t \in\left[\tau_{i_{k}}, \xi_{k+1}\right),\end{cases}
$$

where

$$
\bar{\ell}_{k}:=\min \left\{\underset{\ell \in \overline{1, n_{V}}}{\operatorname{Argmin}} \max _{j \in \overline{0, k}} \frac{1}{\tau_{i_{j}}-\xi_{j}}\left\|\int_{\xi_{j}}^{\tau_{i_{j}}}\left(\bar{g}(t)-g_{\ell}(t)\right) d t\right\|\right\} .
$$

Let us show that, for every $k \in \overline{0, n_{\Delta_{*}}}$, the inequality below is valid:

$$
\left\|\bar{x}(t)-x_{\bar{\ell}}(t)\right\| \leq 2 L k e^{\lambda\left(\xi_{k}-t_{0}\right)} \delta, \quad t \in\left[t_{0}, \xi_{k}\right] .
$$

For $k=0$, we have $\xi_{0}=t_{0}$, and, hence, inequality (3.14) holds due to initial condition (1.2). Arguing by induction, let us take $k_{*} \in \overline{0, n_{\Delta_{*}}-1}$, assume that this inequality is fulfilled for $k=k_{*}$, and prove it for $k=k_{*}+1$.

To this end, let us establish the inequality

$$
s_{\bar{\ell}, \bar{\ell}_{k_{*}}}>\xi_{k_{*}} \text {. }
$$

Observe that, for every $j \in \overline{0, k_{*}}$ and every $t \in\left[\xi_{j}, \tau_{i_{j}}\right]$, by the induction assumption and the choice of $\lambda, L$, and $\delta$, we get

$$
\begin{gathered}
\left\|\bar{g}(t)-g_{\bar{\ell}}(t)\right\| \leq \lambda\left\|\bar{x}(t)-x_{\bar{\ell}}(t)\right\| \leq \\
\leq \lambda\left(\left\|\bar{x}(t)-\bar{x}\left(\xi_{j}\right)\right\|+\left\|\bar{x}\left(\xi_{j}\right)-x_{\bar{\ell}}\left(\xi_{j}\right)\right\|+\left\|x_{\bar{\ell}}\left(\xi_{j}\right)-x_{\bar{\ell}}(t)\right\|\right) \leq \\
\leq \lambda\left(2 L \delta+2 L k_{*} e^{\lambda\left(\xi_{k_{*}}-t_{0}\right)} \delta\right) \leq 2 L \lambda n_{\Delta_{*}} e^{\lambda\left(\vartheta-t_{0}\right)} \delta \leq \zeta / 8 .
\end{gathered}
$$


Hence, and owing to the definition of $\bar{\ell}_{k_{*}}$ as one of the minimizing elements, we conclude that

$$
\max _{j \in \overline{0, k_{*}}} \frac{1}{\tau_{i_{j}}-\xi_{j}}\left\|\int_{\xi_{j}}^{\tau_{i_{j}}}\left(\bar{g}(t)-g_{\bar{\ell}_{k_{*}}}(t)\right) d t\right\| \leq \max _{j \in \overline{0, k_{*}}} \frac{1}{\tau_{i_{j}}-\xi_{j}}\left\|\int_{\xi_{j}}^{\tau_{i_{j}}}\left(\bar{g}(t)-g_{\bar{\ell}}(t)\right) d t\right\| \leq \frac{\zeta}{8} .
$$

On the other hand, let us consider $m \in \overline{1, n_{V}}$ such that $s_{\bar{\ell}, m} \leq \xi_{k_{*}}$. In view of (3.6) and (3.7), let us take $j \in \overline{0, k_{*}}$ for which the equality $\xi_{j}=s_{\bar{\ell}, m}$ is valid. We have

$$
\begin{gathered}
\left\|\int_{\xi_{j}}^{\tau_{i_{j}}}\left(\bar{g}(t)-g_{m}(t)\right) d t\right\| \geq \\
\geq\left\|\int_{\xi_{j}}^{\tau_{i_{j}}}\left(g_{\bar{\ell}}(t)-g_{\bar{\ell}, m}(t)\right) d t\right\|-\left\|\int_{\xi_{j}}^{\tau_{i_{j}}}\left(\bar{g}(t)-g_{\bar{\ell}}(t)\right) d t\right\|-\left\|\int_{\xi_{j}}^{\tau_{i_{j}}}\left(g_{\bar{\ell}, m}(t)-g_{m}(t)\right) d t\right\| .
\end{gathered}
$$

Let us estimate each of the terms from the right-hand side of this inequality. For the first term, due to the choice of $\omega$ and $\delta$, we derive

$$
\left\|\int_{\xi_{j}}^{\tau_{i_{j}}}\left(g_{\bar{\ell}}(t)-g_{\bar{\ell}, m}(t)\right) d t\right\| \geq \frac{\zeta}{2}\left(\tau_{i_{j}}-\xi_{j}\right) .
$$

For the second term, using (3.16), we get

$$
\left\|\int_{\xi_{j}}^{\tau_{i_{j}}}\left(\bar{g}(t)-g_{\bar{\ell}}(t)\right) d t\right\| \leq \frac{\zeta}{8}\left(\tau_{i_{j}}-\xi_{j}\right) .
$$

In order to estimate the third term, we note that $x_{\bar{\ell}}\left(\xi_{j}\right)=x_{m}\left(\xi_{j}\right)$ according to (3.2), and, therefore, for all $t \in\left[\xi_{j}, \tau_{i_{j}}\right]$, we derive

$$
\begin{gathered}
\left\|g_{\bar{\ell}, m}(t)-g_{m}(t)\right\| \leq \lambda\left\|x_{\bar{\ell}}(t)-x_{m}(t)\right\| \leq \\
\leq \lambda\left(\left\|x_{\bar{\ell}}(t)-x_{\bar{\ell}}\left(\xi_{j}\right)\right\|+\left\|x_{m}\left(\xi_{j}\right)-x_{m}(t)\right\|\right) \leq 2 \lambda L \delta \leq \zeta / 8 .
\end{gathered}
$$

Thus, we come to the inequality

$$
\frac{1}{\tau_{i_{j}}-\xi_{j}}\left\|\int_{\xi_{j}}^{\tau_{i_{j}}}\left(\bar{g}(t)-g_{m}(t)\right) d t\right\| \geq \frac{\zeta}{4}
$$

As a result, we conclude that, for any $m \in \overline{1, n_{V}}$, if $s_{\bar{\ell}, m} \leq \xi_{k_{*}}$, then

$$
\max _{j \in \overline{0, k_{*}}} \frac{1}{\tau_{i_{j}}-\xi_{j}}\left\|\int_{\xi_{j}}^{\tau_{i_{j}}}\left(\bar{g}(t)-g_{m}(t)\right) d t\right\| \geq \frac{\zeta}{4} .
$$

This estimate combined with (3.17) yields (3.15).

Further, in view of (3.6) and (3.7), inequality (3.15) implies that $s_{\bar{\ell}, \bar{\ell}_{k_{*}}} \geq \xi_{k_{*}+1}$. Hence, by (3.4), we have $f_{\bar{\ell}}(t)=f_{\bar{\ell}_{,}, \bar{\ell}_{k_{*}}}(t)$ for a. e. $t \in\left[\tau_{i_{k_{*}}}, \xi_{k_{*}+1}\right]$. Respectively, taking (3.13) into account, we obtain

$$
f_{\bar{\ell}}(t)=f\left(t, x_{\bar{\ell}}(t), \bar{u}(t)\right) \quad \text { for a. e. } t \in\left[\tau_{i_{k_{*}}}, \xi_{k_{*}+1}\right) .
$$

Now, based on the induction assumption, for all $t \in\left[\xi_{k_{*}}, \tau_{i_{*}}\right]$, we derive

$$
\begin{aligned}
\left\|\bar{x}(t)-x_{\bar{\ell}}(t)\right\| & \leq\left\|\bar{x}(t)-\bar{x}\left(\xi_{k_{*}}\right)\right\|+\left\|\bar{x}\left(\xi_{k_{*}}\right)-x_{\bar{\ell}}\left(\xi_{k_{*}}\right)\right\|+\left\|x_{\bar{\ell}}\left(\xi_{k_{*}}\right)-x_{\bar{\ell}}(t)\right\| \leq \\
& \leq 2 L \delta+2 L k_{*} e^{\lambda\left(\xi_{k_{*}}-t_{0}\right)} \delta \leq 2 L\left(k_{*}+1\right) e^{\lambda\left(\xi_{k_{*}}-t_{0}\right)} \delta .
\end{aligned}
$$


Due to (3.18) and (3.19), for every $t \in\left[\tau_{i_{* *}}, \xi_{k_{*}+1}\right]$, we have

$$
\begin{gathered}
\left\|\bar{x}(t)-x_{\bar{\ell}}(t)\right\| \leq \\
\leq\left\|\bar{x}\left(\tau_{i_{k_{*}}}\right)-x_{\bar{\ell}}\left(\tau_{i_{k_{*}}}\right)\right\|+\int_{\tau_{i_{k_{*}}}}^{t}\left\|\bar{f}(\tau)-f\left(\tau, x_{\bar{\ell}}(\tau), \bar{u}(\tau)\right)\right\| d \tau+\int_{\tau_{i_{k_{*}}}}^{t}\left\|\bar{g}(\tau)-g_{\bar{\ell}}(\tau)\right\| d \tau \leq \\
\leq 2 L\left(k_{*}+1\right) e^{\lambda\left(\xi_{k_{*}}-t_{0}\right)} \delta+\lambda \int_{\tau_{i_{k *}}}^{t}\left\|\bar{x}(\tau)-x_{\bar{\ell}}(\tau)\right\| d \tau .
\end{gathered}
$$

Consequently, by Gronwall's inequality, for all $t \in\left[\tau_{i_{*}}, \xi_{k_{*}+1}\right]$, we get

$$
\left\|\bar{x}(t)-x_{\bar{\ell}}(t)\right\| \leq 2 L\left(k_{*}+1\right) e^{\lambda\left(\xi_{k_{*}}-t_{0}\right)} \delta e^{\lambda\left(t-\tau_{i_{* *}}\right)} \leq 2 L\left(k_{*}+1\right) e^{\lambda\left(\xi_{k_{*}+1}-t_{0}\right)} \delta .
$$

Inequality (3.14) for $k=k_{*}+1$ follows from the induction assumption and estimates (3.19) and (3.20).

Thus, it remains to note that inequality (3.14) for $k=n_{\Delta_{*}}$ implies inequality (3.12) for $\ell=\bar{\ell}$ owing to the choice of $\delta$. The lemma is proved.

\section{$\S 4$. Main result}

The main result of the paper is the following.

Theorem 1. Suppose that, for every $\varepsilon>0$, there exists a non-anticipative strategy $\alpha_{\varepsilon} \in \mathcal{A}$ for which the property of strict distinguishability is fulfilled and the inequality below is valid:

$$
\max _{\ell \in \overline{1, n_{V}}} \sigma\left(x\left(\cdot ; \alpha_{\varepsilon}\left(v_{\ell}(\cdot)\right)(\cdot), v_{\ell}(\cdot)\right)\right) \leq \Gamma+\varepsilon .
$$

Then, the optimal guaranteed result in the class of non-anticipative strategies coincides with that in the class of full-memory strategies, i.e., the equality $\Gamma=\Gamma_{*}$ holds.

P r o o f. Since $\Gamma \leq \Gamma_{*}$ due to Lemma 1, it suffices to show that $\Gamma_{*} \leq \Gamma$. Let us fix $\varepsilon>0$ and take $\alpha_{\varepsilon} \in \mathcal{A}$ as in the assumption of the theorem.

Note that, according to (1.6) and by the Arzelà-Ascoli theorem, there is a compact set $X \subset$ $\subset C\left(T ; \mathbb{R}^{n}\right)$ such that $x\left(\cdot ; u(\cdot), v_{\ell}(\cdot)\right) \in X$ for any $u(\cdot) \in \mathcal{U}$ and any $\ell \in \overline{1, n_{V}}$. Hence, recalling that the function $\sigma$ is continuous, let us choose $\eta>0$ such that, for every $x(\cdot), y(\cdot) \in X$, if $\|x(t)-y(t)\| \leq \eta$ for all $t \in T$, then $|\sigma(x(\cdot))-\sigma(y(\cdot))| \leq \varepsilon$. Further, by Lemma 2, there exists a full-memory strategy $U:=\left(U^{\Delta}\right)_{\Delta \in \Delta_{T}} \in \mathcal{S}$ and a number $\delta>0$ such that, for any $\Delta \in \Delta_{T}$ satisfying the condition $\mathrm{d}(\Delta) \leq \delta$ and any $\ell \in \overline{1, n_{V}}$, the inequality

$$
\left\|x\left(t ; U^{\Delta}, v_{\ell}(\cdot)\right)-x\left(t ; \alpha_{\varepsilon}\left(v_{\ell}(\cdot)\right)(\cdot), v_{\ell}(\cdot)\right)\right\| \leq \eta, \quad t \in T,
$$

is valid. Then, by virtue of the choice of $\eta$ and inequality (4.1), for every $\Delta \in \Delta_{T}$ such that $\mathrm{d}(\Delta) \leq \delta$ and every $\ell \in \overline{1, n_{V}}$, we obtain

$$
\sigma\left(x\left(\cdot ; U^{\Delta}, v_{\ell}(\cdot)\right)\right) \leq \sigma\left(x\left(\cdot ; \alpha_{\varepsilon}\left(v_{\ell}(\cdot)\right)(\cdot), v_{\ell}(\cdot)\right)\right)+\varepsilon \leq \Gamma+2 \varepsilon .
$$

Consequently, in accordance with definition (2.9) of $\Gamma_{*}$, we derive

$$
\Gamma_{*} \leq \lim _{\delta \rightarrow+0} \sup _{\Delta \in \Delta_{T}: \mathrm{d}(\Delta) \leq \delta} \max _{\ell \in \overline{1, n_{V}}} \sigma\left(x\left(\cdot ; U^{\Delta}, v_{\ell}(\cdot)\right)\right) \leq \Gamma+2 \varepsilon .
$$

Thus, we conclude that $\Gamma_{*} \leq \Gamma+2 \varepsilon$ for any $\varepsilon>0$ and, therefore, $\Gamma_{*} \leq \Gamma$.

In Theorem 1, it is assumed that the property of strict distinguishability is fulfilled. Let us present a sufficient condition for this property to be valid. 
Proposition 1. Assume that the function $Q \ni v \mapsto g(t, x, v) \in \mathbb{R}^{n}$ is injective for every $t \in T$ and every $x \in \mathbb{R}^{n}$ and all disturbances $v_{\ell}(\cdot), \ell \in \overline{1, n_{V}}$, are piecewise constant. Then, the property of strict distinguishability is valid for any non-anticipative strategy $\alpha \in \mathcal{A}$.

P r o o f. Let us fix $\alpha \in \mathcal{A}$ and consider the corresponding system's motions $x_{\ell}(\cdot)$, functions $g_{\ell}(\cdot)$ and $g_{\ell, m}(\cdot)$, and times $s_{\ell, m}$ for all $\ell, m \in \overline{1, n_{V}}$, which are given by (2.1), (2.3), and (3.1).

Let us take $\ell, m \in \overline{1, n_{V}}$ satisfying the condition $s_{\ell, m}<\vartheta$. Since the functions $v_{\ell}(\cdot)$ and $v_{m}(\cdot)$ are piecewise constant, there are $s^{*} \in\left(s_{\ell, m}, \vartheta\right]$ and $v_{\ell}^{*}, v_{m}^{*} \in Q$ such that $v_{\ell}(t)=v_{\ell}^{*}$ and $v_{m}(t)=v_{m}^{*}$ for all $t \in\left(s_{\ell, m}, s^{*}\right]$. Hence, we have

$$
g_{\ell}(t)=g\left(t, x_{\ell}(t), v_{\ell}^{*}\right), \quad g_{\ell, m}(t)=g\left(t, x_{\ell}(t), v_{m}^{*}\right), \quad t \in\left(s_{\ell, m}, s^{*}\right]
$$

and, consequently,

$$
\lim _{t \rightarrow s_{\ell, m}+0}\left\|g_{\ell}(t)-g_{\ell, m}(t)\right\|=\left\|g\left(s_{\ell, m}, x_{\ell}\left(s_{\ell, m}\right), v_{\ell}^{*}\right)-g\left(s_{\ell, m}, x_{\ell}\left(s_{\ell, m}\right), v_{m}^{*}\right)\right\| .
$$

Let us suppose that this limit is equal to 0 . Then, by virtue of the injectivity assumption, we get $v_{\ell}^{*}=v_{m}^{*}$. Therefore, the equality $g_{\ell}(t)=g_{\ell, m}(t)$ holds for all $t \in\left(s_{\ell, m}, s^{*}\right]$, and, thus, we come to a contradiction with the definition of $s_{\ell, m}$. Hence, we conclude that inequality (3.5) takes place, which completes the proof.

\section{§5. Example}

Let us consider the dynamical system

$$
d x(t) / d t=u(t)+v(t), \quad t \in T:=[0,3], \quad x(0)=0,
$$

where $x(t) \in \mathbb{R}$ and the control and disturbance are subject to the instantaneous constraints

$$
u(t) \in P:=[-1,1], \quad v(t) \in Q:=\{-1,0,1\}, \quad t \in T .
$$

Let us suppose that the set $V$ of admissible disturbances consists of two functions

$$
v_{1}(t):=\left\{\begin{array}{ll}
0, & \text { if } t \in[0,1] \cup(2,3], \\
1, & \text { if } t \in(1,2],
\end{array} v_{2}(t):= \begin{cases}0, & \text { if } t \in[0,1], \\
-1, & \text { if } t \in(1,3] .\end{cases}\right.
$$

The aim of the control is to minimize the cost functional

$$
J\left(u(\cdot), v_{\ell}(\cdot)\right):=-\left|x\left(3 ; u(\cdot), v_{\ell}(\cdot)\right)\right|, \quad u(\cdot) \in \mathcal{U}, \quad \ell \in\{1,2\} .
$$

Let us calculate the value $\Gamma$ of the optimal guaranteed result in the class of non-anticipative strategies. To this end, let us first note that, in the considered example, a mapping $\alpha: V \rightarrow \mathcal{U}$ is a non-anticipative strategy if and only if

$$
x\left(t ; \alpha\left(v_{1}(\cdot)\right)(\cdot), v_{1}(\cdot)\right)=x\left(t ; \alpha\left(v_{2}(\cdot)\right)(\cdot), v_{2}(\cdot)\right), \quad t \in[0,1] .
$$

Further, let us define a mapping $\alpha_{0}: V \rightarrow \mathcal{U}$ by

$$
\alpha_{0}\left(v_{1}(\cdot)\right)(t):=\left\{\begin{array}{ll}
0.5, & \text { if } t \in[0,1), \\
1, & \text { if } t \in[1,3],
\end{array} \quad \alpha_{0}\left(v_{2}(\cdot)\right)(t):= \begin{cases}0.5, & \text { if } t \in[0,1), \\
-1, & \text { if } t \in[1,3]\end{cases}\right.
$$

By direct calculations, we get $\alpha_{0} \in \mathcal{A}$ (see (5.2)) and

$$
\max \left\{-\left|x\left(3 ; \alpha_{0}\left(v_{1}(\cdot)\right)(\cdot), v_{1}(\cdot)\right)\right|,-\left|x\left(3 ; \alpha_{0}\left(v_{2}(\cdot)\right)(\cdot), v_{2}(\cdot)\right)\right|\right\}=-3.5 .
$$


Now, if we assume that there is $\alpha \in \mathcal{A}$ such that

$$
\left|x\left(3 ; \alpha\left(v_{1}(\cdot)\right)(\cdot), v_{1}(\cdot)\right)\right|>3.5, \quad\left|x\left(3 ; \alpha\left(v_{2}(\cdot)\right)(\cdot), v_{2}(\cdot)\right)\right|>3.5,
$$

then, due to the instantaneous constraints on the control (see (5.1)), we obtain

$$
x\left(1 ; \alpha\left(v_{1}(\cdot)\right)(\cdot), v_{1}(\cdot)\right)>0.5>x\left(1 ; \alpha\left(v_{2}(\cdot)\right)(\cdot), v_{2}(\cdot)\right),
$$

which contradicts (5.2). Thus, we conclude that $\Gamma=-3.5$.

Theorem 1 and Proposition 1 imply that, in the problem under consideration, the optimal guaranteed result in the class of full-memory strategies is $\Gamma_{*}=-3.5$. Moreover, in accordance with Section 3 and Lemma 2, based on the (optimal) non-anticipative strategy $\alpha_{0}$, defined by (5.3), we can construct a full-memory strategy that provides this result $\Gamma_{*}=-3.5$.

In addition, let us observe that, for the value of the optimal guaranteed result in the class of open-loop controls, we have

$$
\Gamma^{*}:=\inf _{u(\cdot) \in \mathcal{U}} \max \left\{-\left|x\left(3 ; u(\cdot), v_{1}(\cdot)\right)\right|,-\left|x\left(3 ; u(\cdot), v_{2}(\cdot)\right)\right|\right\}=-2>\Gamma_{*},
$$

and an open-loop control achieving the infimum in (5.4) is $u_{0}(t):=-1$ for all $t \in[0,3]$.

Finally, if we pass from the problem with only two admissible disturbances $v_{1}(\cdot)$ and $v_{2}(\cdot)$ to the problem in which every disturbance $v(\cdot) \in \mathcal{V}$ is admissible, then, for the corresponding value of the optimal guaranteed result in the class of full-memory strategies, we get

$$
\widetilde{\Gamma}_{*}:=\inf _{U \in \mathcal{S}} \lim _{\delta \rightarrow+0} \sup _{\Delta \in \Delta_{T}: \mathrm{d}(\Delta) \leq \delta} \sup _{v(\cdot) \in \mathcal{V}}\left(-\left|x\left(3 ; U^{\Delta}, v(\cdot)\right)\right|\right)=0 .
$$

In particular, the infimum in (5.5) is achieved at the full-memory (more precisely, positional) strategy $\widetilde{U}:=\left(\widetilde{U}^{\Delta}\right)_{\Delta \in \Delta_{T}} \in \mathcal{S}$ such that, for every partition $\Delta:=\left(\tau_{i}\right)_{i \in \overline{0, n_{\Delta}}} \in \Delta_{T}$, the full-memory feedback $\widetilde{U}^{\Delta}:=\left(\widetilde{U}_{i}^{\Delta}\right)_{i \in \overline{0, n_{\Delta}-1}}$ is given by

$$
\widetilde{U}_{i}^{\Delta}(x(\cdot))(t):=\left\{\begin{array}{ll}
1, & \text { if } x\left(\tau_{i}\right)>0, \\
0, & \text { if } x\left(\tau_{i}\right)=0, \\
-1, & \text { if } x\left(\tau_{i}\right)<0,
\end{array} \quad t \in\left[\tau_{i}, \tau_{i+1}\right), \quad x(\cdot) \in C\left(\left[t_{0}, \tau_{i}\right] ; \mathbb{R}^{n}\right), \quad i \in \overline{0, n_{\Delta}-1} .\right.
$$

Nevertheless, for any $\Delta \in \Delta_{T}$, we derive

$$
3-\mathrm{d}(\Delta) \leq x\left(3 ; \widetilde{U}^{\Delta}, v_{1}(\cdot)\right) \leq 3, \quad-4 \leq x\left(3 ; \widetilde{U}^{\Delta}, v_{2}(\cdot)\right) \leq-4+\mathrm{d}(\Delta),
$$

and, consequently, in the original problem with the admissible disturbances $v_{1}(\cdot)$ and $v_{2}(\cdot)$, the full-memory strategy $\widetilde{U}$ does not provide the optimal guaranteed result $\Gamma_{*}=-3.5$.

Funding. The work was performed as part of research conducted in the Ural Mathematical Center with the financial support of the Ministry of Science and Higher Education of the Russian Federation (Agreement number 075-02-2021-1383).

\section{REFERENCES}

1. Isaacs R. Differential games, New York: John Wiley and Sons, 1965. https://zbmath.org/?q=an:0125.38001

2. Pontrjagin L. S. Linear differential games of pursuit, Mathematics of the USSR-Sbornik, 1981, vol. 40, no. 3, pp. 285-303. https://doi.org/10.1070/SM1981v040n03ABEH001815

3. Krasovskii N. N., Subbotin A. I. Game-theoretical control problems, New York: Springer, 1988. https://www.springer.com/gp/book/9781461283188 
4. Krasovskii A. N., Krasovskii N. N. Control under lack of information, Boston: Birkhäuser, 1995. https://doi.org/10.1007/978-1-4612-2568-3

5. Kryazhimskii A.V. The problem of optimization of the ensured result: unimprovability of full-memory strategies, Constantin Caratheodory: An International Tribute, Teaneck, New Jersey: World Scientific, 1991, vol. 1, pp. 636-675. https://doi.org/10.1142/9789814350921_0037

6. Serkov D. A. On a dynamic game problem with an indecomposable set of disturbances, Ural Mathematical Journal, 2019, vol. 5, no. 2, pp. 72-79. https://doi.org/10.15826/umj.2019.2.007

7. Gomoyunov M. I., Serkov D. A. Non-anticipative strategies in guarantee optimization problems under functional constraints on disturbances, Vestnik Udmurtskogo Universiteta. Matematika. Mekhanika. Komp'yuternye Nauki, 2020, vol. 30, issue 4, pp. 553-571. https://doi.org/10.35634/vm200402

8. Barron E. N. Differential games with Lipschitz control functions and fixed initial control positions, Journal of Differential Equations, 1977, vol. 26, issue 2, pp. 161-180. https://doi.org/10.1016/0022-0396(77)90188-7

9. Nikol'skij M. S. A crossing problem with possible stop of engine, Differential Equations, 1993, vol. 29, issue 11, pp. 1681-1684. https://zbmath.org/?q=an:0816.90147

10. Ledyaev Yu. S. Program-predictive feedback control for systems with evolving dynamics, IFACPapersOnLine, 2018, vol. 51, issue 32, pp. 723-726. https://doi.org/10.1016/j.ifacol.2018.11.461

11. Ukhobotov V. I. On a control problem under disturbance and possible breakdown, Proceedings of the Steklov Institute of Mathematics, 2019, vol. 307, suppl. 1, pp. 159-171. https://doi.org/10.1134/S0081543819070137

12. Ushakov V.N., Ershov A.A. On recovering of unknown constant parameter by several test controls, Ufa Mathematical Journal, 2020, vol. 12, no. 4, pp. 99-113. https://doi.org/10.13108/2020-12-4-99

13. Roxin E. Axiomatic approach in differential games, Journal of Optimization Theory and Applications, 1969, vol. 3, issue 3, pp. 153-163. https://doi.org/10.1007/BF00929440

14. Elliott R. J., Kalton N. J. The existence of value in differential games of pursuit and evasion, Journal of Differential Equations, 1972, vol. 12, issue 3, pp. 504-523. https://doi.org/10.1016/0022-0396(72)90022-8

15. Serkov D. A. On the unimprovability of full-memory strategies in problems of guaranteed result optimization, Proceedings of the Steklov Institute of Mathematics, 2015, vol. 291, suppl. 1, pp. 157-172. https://doi.org/10.1134/S0081543815090114

16. Vinter R. B. Minimax optimal control, SIAM Journal on Control and Optimization, 2005, vol. 44, issue 3, pp. 939-968. https://doi.org/10.1137/S0363012902415244 
Mikhail Igorevich Gomoyunov, Candidate of Physics and Mathematics, N. N. Krasovskii Institute of Mathematics and Mechanics, Ural Branch of the Russian Academy of Sciences, ul. S. Kovalevskoi, 16, Yekaterinburg, 620108, Russia;

Ural Federal University, ul. Mira, 19, Yekaterinburg, 620002, Russia.

ORCID: https://orcid.org/0000-0002-0871-920X

E-mail: m.i.gomoyunov@gmail.com

Dmitrii Aleksandrovich Serkov, Doctor of Physics and Mathematics, N. N. Krasovskii Institute of Mathematics and Mechanics, Ural Branch of the Russian Academy of Sciences, ul. S. Kovalevskoi, 16, Yekaterinburg, 620108, Russia;

Ural Federal University, ul. Mira, 19, Yekaterinburg, 620002, Russia.

ORCID: https://orcid.org/0000-0003-0628-6217

E-mail: d.a.serkov@gmail.com

Citation: M. I. Gomoyunov, D. A. Serkov. On guarantee optimization in control problem with finite set of disturbances, Vestnik Udmurtskogo Universiteta. Matematika. Mekhanika. Komp'yuternye Nauki, 2021, vol. 31, issue 4, pp. 613-628. 


\section{М. И. Гомоюнов, Д. А. Серков}

\section{Об оптимизации гарантии в задаче управления с конечным множеством помех}

Ключевые слова: управление в условиях помех, оптимальная гарантия, неупреждающая стратегия, стратегия с полной памятью, распознавание помех, неулучшаемость.

УдК 517.977

DOI: $10.35634 / \mathrm{vm} 210406$

В статье изучается задача управления в условиях помех, которая формулируется как задача оптимизации гарантированного результата. В отличие от классической постановки таких задач предполагается, что множество допустимых помех конечно и состоит из кусочно-непрерывных функций. С учетом этого дополнительного функционального ограничения на помеху определяется подходящий класс неупреждающих стратегий (квазистратегий) управления и рассматривается соответствующая величина оптимального гарантированного результата. При некотором техническом предположении о свойстве различимости допустимых помех доказывается, что этот результат может быть достигнут путем использования стратегий управления с полной памятью. Как следствие, устанавливается неулучшаемость класса стратегий с полной памятью. Ключевым элементом доказательства является процедура распознавания действующих в системе помех, которая позволяет всякой неупреждающей стратегии поставить в соответствие близкую по гарантированному результату стратегию с полной памятью. В заключение статьи приводится иллюстрирующий пример.

Финансирование. Работа выполнена в рамках исследований, проводимых в Уральском математическом центре при финансовой поддержке Министерства науки и высшего образования Российской Федерации (номер соглашения 075-02-2021-1383).

\section{СПИСОК ЛИТЕРАТУРЫ}

1. Айзекс Р. Дифференциальные игры. М.: Мир, 1967.

2. Понтрягин Л. С. Линейные дифференциальные игры преследования // Математический сборник. 1980. T. 112 (154). № 3 (7). C. 307-330. http://mi.mathnet.ru/msb2728

3. Красовский Н. Н., Субботин А. И. Позиционные дифференциальные игры. М.: Наука, 1974.

4. Krasovskii A. N., Krasovskii N. N. Control under lack of information. Boston: Birkhäuser, 1995. https://doi.org/10.1007/978-1-4612-2568-3

5. Kryazhimskij A. V. The problem of optimization of the ensured result: unimprovability of full-memory strategies // Constantin Caratheodory: An International Tribute. Teaneck, New Jersey: World Scientific, 1991. Vol. 1. P. 636-675. https://doi.org/10.1142/9789814350921_0037

6. Serkov D. A. On a dynamic game problem with an indecomposable set of disturbances // Ural Mathematical Journal. 2019. Vol. 5. No. 2. P. 72-79. https://doi.org/10.15826/umj.2019.2.007

7. Гомоюнов М. И., Серков Д. А. Неупреждающие стратегии в задачах оптимизации гарантии при функциональных ограничениях на помехи // Вестник Удмуртского университета. Математика. Механика. Компьютерные науки. 2020. Т. 30. Вып. 4. С. 553-571. https://doi.org/10.35634/vm200402

8. Barron E. N. Differential games with Lipschitz control functions and fixed initial control positions // Journal of Differential Equations. 1977. Vol. 26. Issue 2. P. 161-180. https://doi.org/10.1016/0022-0396(77)90188-7

9. Никольский М. С. Задача о переправе с возможной остановкой двигателя // Дифференциальные уравнения. 1993. Т. 29. № 11. С. 1937-1940. http://mi.mathnet.ru/de8235

10. Ledyaev Yu. S. Program-predictive feedback control for systems with evolving dynamics // IFACPapersOnLine. 2018. Vol. 51. Issue 32. P. 723-726. https://doi.org/10.1016/j.ifacol.2018.11.461

11. Ухоботов В. И. Об одной задаче управления при наличии помехи и возможной поломке // Труды Института математики и механики УрО РАН. 2019. Т. 25. № 3. С. 265-278.

https://doi.org/10.21538/0134-4889-2019-25-3-265-278 
12. Ушаков В.Н., Ершов А. А. О восстановлении неопределенного постоянного параметра несколькими пробными управлениями // Уфимский математический журнал. 2020. Т. 12. № 4. C. 101-116. http://mi.mathnet.ru/ufa540

13. Roxin E. Axiomatic approach in differential games // Journal of Optimization Theory and Applications. 1969. Vol. 3. Issue 3. P. 153-163. https://doi.org/10.1007/BF00929440

14. Elliott R. J., Kalton N. J. The existence of value in differential games of pursuit and evasion // Journal of Differential Equations. 1972. Vol. 12. Issue 3. P. 504-523.

https://doi.org/10.1016/0022-0396(72)90022-8

15. Серков Д. А. О неулучшаемости стратегий с полной памятью в задачах оптимизации гарантированного результата // Труды Института математики и механики УрО РАН. 2014. Т. 20. № 3. C. 204-217. http://mi.mathnet.ru/timm1095

16. Vinter R. B. Minimax optimal control // SIAM Journal on Control and Optimization. 2005. Vol. 44. Issue 3. P. 939-968. https://doi.org/10.1137/S0363012902415244

Поступила в редакцию 04.10 .2021

Гомоюнов Михаил Игоревич, к. ф.-м. н., старший научный сотрудник, Институт математики и механики им. Н. Н. Красовского УрО РАН, 620108, Россия, г. Екатеринбург, ул. С. Ковалевской, 16;

доцент, ИЕНиМ, Уральский федеральный университет им. Б. Н. Ельцина, 620002, Россия, г. Екатеринбург, ул. Мира, 19.

ORCID: https://orcid.org/0000-0002-0871-920X

E-mail: m.i.gomoyunov@gmail.com

Серков Дмитрий Александрович, д.ф.-м.н., зав. сектором, Институт математики и механики им. Н. Н. Красовского УрО РАН, 620108, Россия, г. Екатеринбург, ул. С. Ковалевской, 16;

профессор, ИРИТ-РТФ, Уральский федеральный университет им. Б.Н. Ельцина, 620002, Россия, г. Екатеринбург, ул. Мира, 19.

ORCID: https://orcid.org/0000-0003-0628-6217

E-mail: d.a.serkov@gmail.com

Цитирование: М. И. Гомоюнов, Д. А. Серков. Об оптимизации гарантии в задаче управления с конечным множеством помех // Вестник Удмуртского университета. Математика. Механика. Компьютерные науки. 2021. Т. 31. Вып. 4. С. 613-628. 